\title{
Type 1 diabetes mellitus: much progress, many opportunities
}

\author{
Alvin C. Powers \\ Division of Diabetes, Endocrinology and Metabolism, Department of Medicine, Vanderbilt University Medical Center, Nashville, Tennessee, USA. Department of Molecular Physiology and Biophysics, \\ Vanderbilt University School of Medicine, Nashville, Tennessee, USA. VA Tennessee Valley Healthcare System, Nashville, Tennessee, USA.

\begin{abstract}
As part of the centennial celebration of insulin's discovery, this review summarizes the current understanding of the genetics, pathogenesis, treatment, and outcomes in type 1 diabetes (T1D). T1D results from an autoimmune response that leads to destruction of the $\beta$ cells in the pancreatic islet and requires lifelong insulin therapy. While much has been learned about T1D, it is now clear that there is considerable heterogeneity in T1D with regard to genetics, pathology, response to immune-based therapies, clinical course, and susceptibility to diabetes-related complications. This Review highlights knowledge gaps and opportunities to improve the understanding of T1D pathogenesis and outlines emerging therapies to treat or prevent T1D and reduce the burden of $\mathrm{T} 1 \mathrm{D}$.
\end{abstract}

\section{Introduction}

The discovery of insulin and its rapid incorporation into clinical practice is one the greatest examples of scientific research saving lives and transforming clinical care. Prior to the discovery of insulin, type 1 diabetes mellitus (T1D) was a uniformly lethal disease that led to either rapid death from diabetic ketoacidosis or, with adherence to a strict starvation protocol, severe malnutrition and death within months or 1-2 years. As outlined by historians (1), the discovery of insulin was a reprieve from the death sentence of T1D, such that in 1922-1923, the timing of T1D onset and access to insulin determined whether some individuals died or lived for many years (2). The stories of people rescued from certain death by insulin's discovery are nothing short of miraculous. Multiple millions of individuals with T1D and their descendants are alive today because of insulin's discovery.

This Review provides a brief, comprehensive overview of human T1D, including what is known about its genetics, pathogenesis, and natural history, before concluding with a discussion of current and future therapeutic or preventive strategies. This Review describes exciting advances in the understanding and treatment of human T1D, but also highlights gaps in our knowledge that must be overcome to reverse and/or prevent T1D and its related complications. A recurring theme will be the growing recognition that T1D is not a single, monolithic disease but is instead heterogenous, and that the term T1D likely encompasses several different pathological processes within the clinical phenotype of T1D. While this Review is aimed at medical and scientific readers, it is essential to recognize the critical roles played by individuals with T1D and their families in advancing the understanding and treatment of T1D. These individuals have participated in paradigm-changing clinical trials, advocated for biomedical research

Conflict of interest: The author has declared that no conflict of interest exists. Copyright: () 2021, American Society for Clinical Investigation.

Reference information: J Clin Invest. 2021;131(8):e142242.

https://doi.org/10.1172/JCl142242. and new technologies, and incorporated many new approaches into their daily lives.

\section{Diagnosis of T1D in 2021}

Traditionally, the diagnosis of T1D is based on the clinical phenotype of insulin-dependent diabetes with onset in childhood or adolescence and possibly diabetic ketoacidosis. With the increasing emphasis on precision medicine, most discussion of diabetes has focused on heterogeneity in type 2 diabetes (T2D) and distinguishing T2D from monogenic diabetes or atypical forms of diabetes (3-5), with the assumption that one can accurately define T1D based on the clinical phenotype. This view has been substantially altered by two major changes in our thinking: (a) T1D begins with evidence of islet-directed autoimmunity prior to appearance of dysglycemia or hyperglycemia; (b) T1D likely results from different pathways to $\beta$ cell destruction as reflected by differences in age of onset, genetics, pancreas pathology, metabolism, insulin secretion, and ultimately by differences in response to therapies and diabetes-related complications.

The model proposed by George Eisenbarth in 1986 has long been the standard schematic for how T1D develops, and even with many noted caveats and inconsistencies, it remains useful in framing today's discussion about the development of T1D (Figure 1 and Table 1). Components, revisions, and limitations of this model are discussed below and include new information about genetic susceptibility, pathogenic and molecular aspects of $\beta$ cell-directed autoimmunity, and the interactions between immunology and islet biology (6). The current model suggests that the natural progression of T1D in genetically susceptible individuals consists of three stages (7). In stage 1 , normoglycemia is accompanied by two or more islet-directed autoantibodies. The autoimmune process, as reflected by these autoantibodies, is presumably initiated by an unidentified triggering event or events. In stage 2 , the autoantibodies are accompanied by dysglycemia reflecting inadequate insulin secretion after a glucose or nutrient challenge. In stage 3, the time that T1D is usually clinically diagnosed, symptoms are 


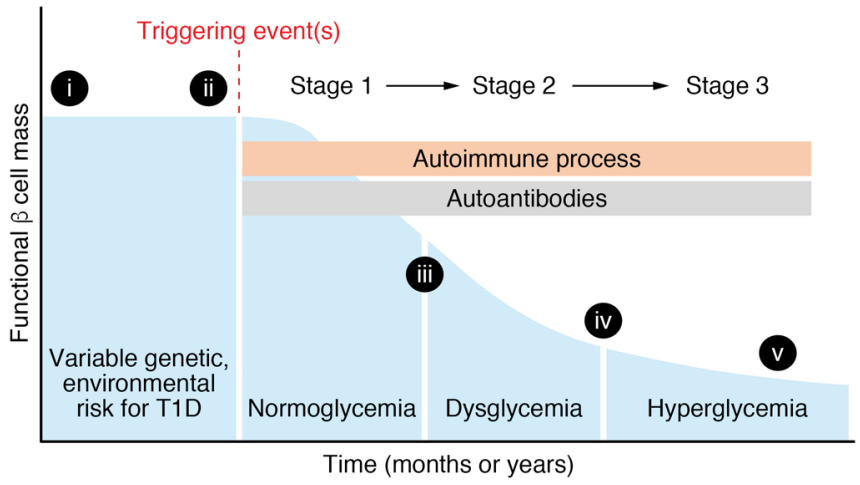

Figure 1. Model of stages of type 1 diabetes (T1D). Graph shows functional $\beta$ cell mass through the stages of T1D. The blue shaded area shows number or insulin secretory capacity of $\beta$ cells, with time on the $x$ axis reflecting a broad range (could be months or years of T1D development). See text for definition of T1D stages. Roman numerals on the graph refer to questions about T1D pathogenesis shown in Table 1.

usually present and insulin therapy is initiated. Presentation may range from diabetic ketoacidosis to modest hyperglycemia. $\beta$ cells are still present and functional (it is estimated that $\sim 60 \%-90 \%$ of $\beta$ cell mass has been lost at clinical presentation; ref. 8 ). $\beta$ Cell loss continues over the ensuing months to years, but many individuals with longstanding T1D still secrete small amounts of insulin. Although these stages are a helpful framework, it should be noted that not all individuals in stage 1 or stage 2 progress to hyperglycemia requiring insulin treatment. A significant limitation of the model is that it is almost entirely based on measurements gathered from the peripheral blood (insulin secretion, immunological markers, etc.) and clinical parameters (age of onset, BMI, exogenous insulin requirements, etc.); only recently have there been studies of the "scene of the crime," the human pancreas, in stages 1, 2, and 3 and recent-onset T1D (discussed below).

There is a lack of accepted, specific criteria for diagnosis of T1D. Instead, the clinical diagnosis continues to rely on two main features: (a) insulin deficiency and need for exogenous insulin therapy (insulin requirements may be modest in early stage 3 and the first years after hyperglycemia onset) and (b) presence of islet-directed autoantibodies. These criteria are reasonably accurate in individuals who develop diabetes prior to 20 years of age, but are considerably less informative in those over the age of 20 years, highlighting the need for additional criteria (e.g., genetic risk score, other immunological makers, subtypes of T1D). Other autoimmune endocrinopathies (autoimmune thyroid disease, autoimmune adrenal insufficiency, celiac disease, or atrophic gastritis) may be present in up to $20 \%$ of individuals with T1D and should be considered (9).

\section{Scope and incidence of T1D}

The precise number of individuals with T1D is not known, but more than 1.6 million people in the United States are thought to have T1D (10). The incidence of T1D varies widely among countries, but this likely reflects differences in T1D-susceptibility genetic loci in a country's population rather than environmental exposures. T1D incidence is highest in Finland, Sardinia, Sweden, Norway, and Portugal, with the rate being 60- to 300-fold greater

\section{Table 1. Questions about current model of T1D development}

i. How does peak $\beta$ cell mass, which varies between individuals, influence susceptibility to T1D-related $\beta$ cell loss? What is $\beta$ cell mass at each stage of T1D?

ii. What triggering event or events initiate $\beta$ cell-directed autoimmunity? Is the triggering event the same in all individuals?

iii. Is $\beta$ cell-directed autoimmunity sustained or does it wax and wane? Is the autoimmunity the same at all stages of T1D?

iv. Is $\beta$ cell loss continuous/progressive or does it start and then stop?

v. Why do some $\beta$ cells survive and others do not? Can remaining $\beta$ cells regenerate?

Question numbers correspond to numbers in Figure 1.

than in China and Venezuela (11-13). The incidence in the United States and Europe is intermediate. These rates of T1D are likely an underestimate and do not include many young adults and adults who develop T1D because almost all incidence data comes from registries with individuals under 20 years of age. The SEARCH for Diabetes in Youth study in the United States found a 1.4\% per year increase in T1D incidence from 2002 to 2012, with an unexpected increase in Hispanic youths (14). This increase in the United States is similar to the gradual worldwide annual increase in T1D incidence over the past 30 years. The reasons for this increase are not known, but may reflect changing diet and environmental exposures. There are some signs, however, that this increase is not a continuing trend (13). The number of individuals developing T1D who have the high-risk HLA alleles is declining (15), suggesting that gene-environment interactions such as diet or environmental exposure play a role.

\section{Genetics of T1D}

A genetic susceptibility to T1D, although incompletely defined, is clear: identical twins have a concordance rate of $60 \%-70 \%$ with long-term follow-up (16) and first-degree relatives have a 5\%-6\% lifetime risk of T1D. Despite the clear genetic risk, most individuals with T1D have no family history of T1D. Approximately $50 \%$ of the genetic risk is related to class II HLA alleles (DR, DQ, and others). Loci related to the insulin gene and variable number tandem repeat convey the next highest genetic risk $(15,17)$. At least 50 other genetic loci or SNPs, mostly in regions of the genome predicted to be involved in gene regulation, have been identified as providing a small risk for T1D. Until recently, these loci were thought to affect immune cell function and influence nearby genes, but new data suggest that some directly affect the $\beta$ cell, perhaps altering $\beta$ cell response to inflammation or cytokines, such as IFN- $\gamma$ or IL-1 $\beta$, or act by influencing distant regulatory regions (17).

The polygenic nature of T1D genetic susceptibility has stimulated efforts to combine SNPs into a T1D genetic risk score (T1DGRS). Such a T1D-GRS has proven useful when combined with autoantibody measurements in enhancing prediction of future T1D development in individuals at high risk for T1D who are being followed in The Environmental Determinants of Diabetes in the Young (TEDDY) study (18). Similarly, application of such a T1DGRS to the UK Biobank predicted that as many as $42 \%$ of individuals with T1D developed T1D over 30 years of age, highlighting the heterogeneity in T1D and the difficulty of diagnosing T1D in 


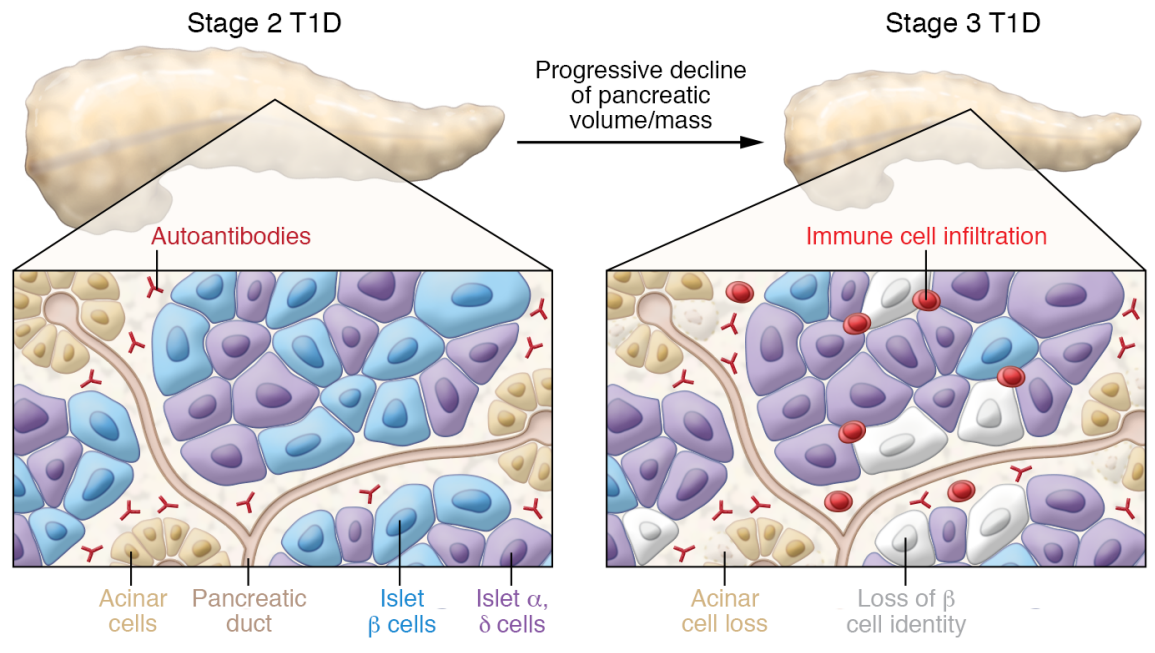

Figure 2. Pancreatic changes and immune abnormalities in T1D. The reduction of pancreas size or volume from normal to stage 2 to stage 3 T1D is shown at the top of the figure. The insets below shows a stylized section of the pancreas with islets and acinar cells in stage 2 and stage 3 T1D. Circulating autoantibodies directed at islet-enriched molecules are present in stage 2 and stage 3 but are not cytotoxic. In stage 3 , immune cells are present within islets and exocrine pancreas, and there is a loss of both $\beta$ cells and acinar cells. Other changes (not shown) in the stage 3 T1D islets include (a) insulin-negative, pseudoatrophic islets with rare islets appearing normal or having $\beta$ cells; (b) alterations in proinsulin and insulin processing and expression of islet-enriched transcription factors such as PDX-1 and NKX6.1; (c) islet cell hyperexpression of HLA class I and class II molecules; (d) insulitis (immune cell infiltration in some islets) is variable, involving primarily CD8+ $\mathrm{T}$ cells, but also B lymphocytes, CD4+ T Iymphocytes, and macrophages; CD20+ B lymphocytes are more common in recent-onset T1D in younger individuals; and (e) $\beta$ cell mass is variable in stage 1, 2, and 3 (see Figure 1). Changes in the exocrine pancreas include (a) reduced pancreatic volume/mass at T1D onset and in autoantibody-positive individuals; progressive decline in pancreas volume in first 5 years of T1D; (b) acinar cell loss, some exocrine fibrosis in stage 3 pancreas; and (c) leukocyte infiltration of exocrine compartment. See text for additional details.

the adult population where T2D is much more frequent (19). For now, T1D-GRS is a research tool, but with further refinements it may prove useful in supporting or refuting the diagnosis of T1D in an individual or in population screening to identify individuals at high risk for developing T1D.

\section{Pathogenesis of T1D - immune and islet perspectives}

The pathological features of T1D, although often definitively stated (autoimmune, $\mathrm{T}$ cell-mediated $\beta$ cell destruction accompanied by nonpathogenic, islet-directed autoantibodies), in reality has many unknowns, unproven assumptions, and knowledge gaps (Figure 1 and Table 1). It is widely assumed that a triggering event or events initiate the $\beta$ cell-directed autoimmunity, but these events or events remain unknown despite sustained efforts over many years. Many environmental agents have been postulated, including virus infection (Coxsackie, rubella, enterovirus, etc.), diet, intestinal microbiota, cleanliness of the environment, and gene-environmental interactions (20-24). The search continues in the TEDDY study, which is prospectively and systematically investigating potential environmental triggers.

Studies by a number of investigators have provided critical insight by collecting and studying the human pancreas from the few individuals who die at T1D onset, after a relatively short duration of T1D ( $<5$ years), or who were found to have islet- related autoantibodies at the time of organ donation (25-35). Some of the pathological findings in the pancreas in recent-onset T1D include modest, patchy, variable insulitis (immune cell infiltration of islet); some pseudoatrophic, insulin-negative islets; and some islets with normal-appearing $\beta$ cells (Figure 2). The clinical features of T1D (age of diabetes diagnosis, BMI) and biomarkers (autoantibodies, C-peptide, T1D-GRS) mostly correlated with these pathological features of T1D (29), thus providing an important connection between peripheral measurements and pancreas pathology.

$\beta$ Cell-directed autoimmunity involves humoral and cell-mediated autoimmunity (Figure 2). Some combination of autoantibodies against glutamic acid decarboxylase (GAD), insulin (IAA), insulinoma-associated antigen-2 (IA-2), zinc transporter 8 (ZnT8), or tetraspanin-7 (Tspan7) are present at the onset of hyperglycemia in more than $90 \%$ of individuals with the typical T1D phenotype. These nonpathogenic autoantibodies are viewed as markers of the autoimmune process, with the presence of multiple autoantibodies during stage 2 in younger individuals predictive of transition to stage 3 over a 5 - to 10 -year timeline (36). In the TEDDY study that enrolled and followed from birth individuals genetically at high risk for T1D, insulin and GAD autoantibodies appeared in the first 5 years of life, sometimes within the first year of life, and the presence of 2 or more autoantibodies was associated with $60 \%-80 \%$ T1D development in follow-up over $10-15$ years $(37,38)$. The appearance of these autoantibodies is at a time of considerable change and plasticity in human islet morphology, cell composition, and maturation and immune system maturation and establishment, raising the possibility that developmental processes contribute to initiation of $\beta$ cell-directed autoimmunity.

Despite the assumption that $\beta$ cell destruction in T1D is mediated by $\mathrm{T}$ cells, it has been difficult to develop robust, standardized $\mathrm{T}$ cell-based assays (39). One reason is the low frequency of the diabetogenic $\mathrm{T}$ cells in the peripheral circulation of individuals with recent-onset T1D (stages 2 and 3) and the assumption that the pathogenic $\mathrm{T}$ cells of interest are within the islet or pancreas-draining lymph nodes. Recent work has validated this view with the isolation and cloning of $\mathrm{CD}^{+}$and $\mathrm{CD} 8^{+} \mathrm{T}$ cell lines from T1D islets and pancreatic lymph nodes that react to a range of islet antigens (including insulin, GAD, islet-specific glucose-6-phosphatase catalytic subunit-related protein, islet-associated amyloid polypeptide) and, interestingly, also to posttranslationally modified peptides or neoantigens (hybrid insulin peptides, etc.) $(26,28$, $40,41)$. There is an increasing awareness that $\mathrm{B}$ lymphocytes are also involved in the autoimmune process (42).

$\beta$ Cells exist within the human islet, a complex miniorgan (43), and according to current thinking, the $\beta$ cell is not an innocent 


\section{Table 2. Examples of T1D heterogeneity}

$\begin{array}{ll}\text { Clinical } & \text { - Age of onset of } \beta \text { cell-directed autoimmunity and hyperglycemia (from } 6 \text { months to adult); up to } \\ & 40 \% \text { onset in individuals over } 30 \text { years old } \\ & \text { - Other autoimmune endocrinopathies or diseases (thyroid, adrenal, celiac, etc.) in some } \\ & \text { - Degree and rate of development of diabetes-related complications (e.g., retinopathy, nephropathy, } \\ & \text { neuropathy) } \\ \text { Cenetic } & \text { - Presence of HLA DR3/DR4 haplotypes } \\ & \text { - More than } 50 \text { genetic loci } \\ \text { Immunological } & \text { - Autoantibody frequency, profile, and target epitopes } \\ & \text { - Type 1 IFN, innate immunity, and T cell signatures } \\ \text { Metabolic } & \text { - Rate of decline in } \beta \text { cell function and mass in stages } 2 \text { and 3 } \\ & \text { - Degree and duration of residual C-peptide production } \\ & \text { - Response to immunomodulatory therapy } \\ \text { Pathological } & \text { - Insulitis (degree, location) differs between and within individuals } \\ & \text { - CD20+ B lymphocytes in younger-onset T1D } \\ & \text { - Degree of } \beta \text { cell loss } \\ & \text { - Differences between insulin-negative islets and islets with } \beta \text { cells }\end{array}$

Adapted with permission from Battaglia et al. (70) and references therein. found that pancreas size by MRI is reduced at the time of T1D onset and in stages 1, 2, and $3(30,61-64)$. Since islets represent only $1 \%-2 \%$ of the pancreas, the exocrine compartment of the pancreas must also be affected in T1D (Figure 2). Prior studies of pancreas size in T1D were conflicting, likely because they were cross-sectional autopsy studies or utilized a single noninvasive imaging session (ultrasound, computerized tomography, or MRI) that made it difficult to adequately account for the normal variation in pancreas size among individuals. Recent efforts, enabled by improvements in pancreas imaging and the availability of postmortem T1D human pancreata for study, have provided new information. For example, two MRI studies showed that pancreas volume is reduced at the time of clinical T1D onset, that it declines further in the first year after onset, and that it is reduced

bystander simply targeted by a misdirected autoimmune process, but instead the $\beta$ cell is an active participant either in the initiation or acceleration of the process leading to its own death $(6,44-46)$. For example, the $\beta$ cell's extremely high rate of insulin biosynthesis and protein processing has been proposed to render it more susceptible to ER stress and the unfolded protein response, especially in the setting of cytokines released by infiltrating immune cells (47). Altered proinsulin processing in the pancreas and in the peripheral blood in T1D, including preclinical stages, has been noted and correlated with immune markers and markers of $\beta$ cell stress $(35,48-51)$. Multiplexed imaging mass cytometry studies of pancreatic samples collected by the Network for Pancreatic Organ donors with Diabetes (52) or the Human Pancreas Analysis Program (53) showed that at the time insulitis developed, some $\beta$ cells had lost or changed expression of markers of $\beta$ cell identity (C-peptide, PDX-1, NKX6.1), perhaps as a response or adaptation to immune attack $(54,55)$. Perhaps portending the clinical heterogeneity of T1D, there was considerable variability in insulitis, $\beta$ cell number, and gene expression within and between T1D pancreatic samples. Surprisingly, many islets in recent-onset T1D still had normal appearing $\beta$ cells, consistent with observations that individuals with longstanding diabetes secrete small amounts of insulin and that the T1D pancreas continues to harbor insulin-positive cells that are glucose responsive (35, 56-59). Although impaired glucagon and catecholamine secretion in response to hypoglycemia is a feature of T1D (especially longer duration T1D) and is thought to reflect autonomic neuropathy (60), recent information suggests that impaired $\alpha$ cell function with decreased expression of markers of cell identity (ARX) and ectopic expression of NKX6.1 in T1D islets also plays a role $(55,59)$. It is not known whether the impaired $\alpha$ cell function is part of the T1D process or secondary to loss of $\beta$ cell contact with $\alpha$ cells.

\section{Exocrine pancreas in T1D}

In T1D there is not only loss of $\beta$ cells but the entire pancreas is smaller in individuals with longstanding T1D. More recently it has been in autoantibody-positive individuals in stage 2 and in some firstdegree T1D relatives $(62,64)$. Pancreas size does not correlate with T1D duration, and it appears that the decline in pancreas size mostly occurs in the 5 years after clinical T1D onset (65).

The molecular mechanism responsible for the reduced volume is not clear, but there is reduced acinar cell number and evidence of fibrosis $(61,65)$. Loss of an islet-derived trophic factor such as insulin has been proposed. T cell infiltration, including some $\mathrm{T}$ cells that recognize proinsulin, have been noted in the T1D exocrine pancreas $(66,67)$. However, it is unclear how changes in the exocrine pancreas are temporally or mechanistically related to $\beta$ cell loss or clinical T1D onset. One hypothesis is that the process affecting the exocrine compartment leads ultimately to $\beta$ cell loss $(68,69)$. How the reduced pancreas volume affects exocrine function in individuals with T1D is not well defined, but reduced pancreatic function (fecal elastase) and pancreatic exocrine insufficiency have been reported in some individuals with T1D $(61,63)$.

\section{Heterogeneity of T1D pathogenic processes and onset}

There is emerging consensus that considerable heterogeneity exists on many levels within the clinical phenotype of T1D $(3,70)$. T1D heterogeneity should not be confused with other causes of insulin deficiency such as certain forms of monogenic diabetes, immune checkpoint-related diabetes, or rare monogenic causes of immune-related diabetes (71-75) that can clinically mimic T1D, but instead refers to clinical, immunological, metabolic, and/or pathological heterogeneity in individuals when the clinical diagnosis of T1D seems likely (Table 2). For example, there is heterogeneity in age of onset, rate of disease progression (decline in $\beta$ cell function and mass), residual C-peptide production (27, 36, 37, 50, 76-79), a spectrum of immunological signatures (autoantibody, $\mathrm{T}$ cell signatures, innate immunity, etc.; refs. 80-84), and pathological abnormalities $(29,31,49,85,86)$. Residual $\beta$ cells, as reflected by low levels of C-peptide, persist for years after onset of hyperglycemia in some individuals with $\operatorname{T1D}(35,56,57)$. Since it is not 
currently possible to noninvasively assess $\beta$ cell mass by imaging or biomarkers other than C-peptide, defining the natural history of $\beta$ cell loss or the impact of interventions to sustain or improve $\beta$ cell mass is problematic. For example, although the decline in $\beta$ cell mass in Figure 1 is shown as a gradual, smooth decline, this is mostly speculative and it is possible that the decline may stop and restart, may stop and remain flat, etc. Using a nomenclature from other diseases with clinical heterogeneity such as asthma, some have proposed that T1D consists of multiple "endotypes," meaning different underlying pathogenic processes that produce a similar phenotype (Table 2) (70).

Two other examples of this heterogeneity are adult-onset T1D (onset after 20 years of age) and a form of diabetes sometimes termed latent autoimmune diabetes in adults (LADA). In the former, individuals have similar phenotypic features to T1D with onset in childhood or adolescence (non-obese, islet-directed autoantibodies, insulin deficiency, glycemic lability, etc.), but fewer $\mathrm{CD} 2 \mathrm{O}^{+} \mathrm{B}$ lymphocytes in the few pancreata that have been studied, and a presumably slower loss of $\beta$ cell mass. In contrast, LADA is often clinically confused with T2D, with onset in middle age, obesity, initial insulin independence then progressing to insulin-dependence, and presence of autoantibodies (often autoantibodies only to GAD in distinction to T1D of childhood or adolescent onset) $(87,88)$. The genetics of LADA show similarities, but not complete overlap, with genetic loci associated with T1D and in some series, the T2D genetic risk loci TCF7L2 is linked $(89,90)$. Whether this variability in genetic loci reflects imprecise phenotyping and inclusion of individuals with T2D is not clear. Critical unanswered questions are whether T1D with onset in the first or second decade of life is the same disease as T1D with onset in older adolescents or young adults, and how these relate to LADA with its onset in middle age.

\section{Current therapy for T1D}

Current T1D therapy focuses on matching exogenous insulin and food intake while incorporating daily activities such as exercise and sleep. Remarkable advances have been made in insulin formulation and diabetes technology, including methods for insulin delivery and glucose monitoring (91-97). Additionally, T1D clinical care is evolving to incorporate mobile technology (smart phones, wearable devices, telemedicine, etc.; ref. 91) and provide greater emphasis on behavioral and psychosocial aspects, the social determinants of health, and health care access/cost. The goal is near normoglycemia while avoiding hypoglycemia and allowing for normal daily activities. Traditionally, the glycemic goal in T1D has been an A1C of 7.0 or lower, with this target individualized for age, comorbidities, and lifestyle (98). Importantly, hypoglycemia, a major adverse effect of intensive glycemic control, is a substantial, lifelong burden of current therapy for T1D (99).

The foundation of insulin therapy for T1D is a combination of basal insulin and bolus insulin associated with nutrient or caloric consumption. Insulin can be given by syringe, pen (including "smart" pens), catheter connected to insulin pump or an insulin delivery device (continuous subcutaneous insulin injection), or more rarely by inhalation $(92,94,100)$. By manipulating the insulin amino acid sequence or by incorporating attachments to the insulin protein (e.g., fatty acid, additional amino acids), the absorption profile of injected insulin can be greatly prolonged or accelerated compared with native insulin protein in a neutral buffer. In addition, insulin diluents can be used to delay absorption (such as protamine in neutral protamine Hagedorn plus native insulin) or accelerate absorption ( $\mathrm{L}$-arginine and niacinamide plus insulin aspart analog) (92). Because of the importance of basal insulin in regulating hepatic glucose output, basal insulin formulations with action duration of more than 40 hours or up to a week have recently become available $(92,101)$. An advantage of longeracting formulations is reduced frequency of severe hypoglycemia. Based on decades of research to understand insulin structure and function (102-104), future possibilities include glucose-responsive insulin (105). The price of insulin formulations in the United States has risen dramatically over the past decade, placing a tremendous financial burden on individuals with T1D $(106,107)$.

These remarkable advances in insulin formulations and insulin delivery devices have greatly improved clinical care. However, many individuals with T1D state that continuous glucose monitoring (CGM) has had an even greater and more dramatic impact on their daily lives. Individuals with T1D for more than 40 years have lived through dramatic transitions from using urine glucose to infer blood glucose over past hours, to finger-stick capillary blood glucose measurements at multiple times a day, to CGM providing an essentially unlimited number of glucose values a day. Although A1C remains the standard for diabetes diagnosis and a predictor of diabetes-related complications, CGM and its dense glycemic datasets allow glycemic metrics, such as the ambulatory glucose profile using time in a defined glycemic range (TIR), the glucose management indicator (GMI) based on the mean glucose and correlative with A1C, glycemic variability or coefficient of variation, and the amount of time in the hypoglycemic range $(98,108)$. These metrics correlate with A1C, are changing how the provider and patient adjust insulin, and are empowering individuals with T1D with information to better organize their daily living routines.

The rapidly evolving CGM technology is currently based on a sensor or electrode detecting the electrochemical product (e.g., hydrogen peroxide) of the reaction between interstitial glucose and a glucose oxidase. Importantly, all current CGM technologies measure interstitial glucose, which, while in equilibrium with blood glucose, may lag behind or differ from blood glucose, especially when the blood glucose is changing rapidly (98, 108-110). Currently, the two broad categories of CGM are real-time CGM, in which interstitial glucose is monitored and sent to the recording device essentially continuously, and intermittent CGM, in which the sensor is in place continuously, but the glucose is only recorded when the detector is placed over the sensor. Both approaches use a subcutaneously placed sensor that must be replaced every 3-14 days; an implanted sensor that must be replaced every 6 months is also available. Although the TIR and GMI are critical CGM outputs, other features such as rate of glucose change, glucose trends, hypoglycemia alarms, and suspension of insulin delivery are extremely valuable, enabling the individual with T1D to respond preemptively and avoid anticipated hyper-or hypoglycemia and to adapt diabetes self-management in terms of diet or activities such as exercise. The pairing of CGM and an insulin delivery device or a closed loop system where the sensor-derived data regulate insulin delivery is discussed below. 
A Exogenous insulin replacement

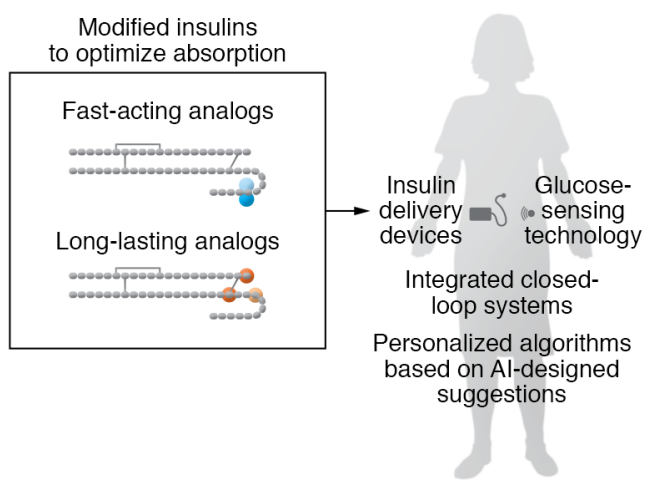

B Cell-based insulin delivery

Transplantation of islets or insulinproducing cells

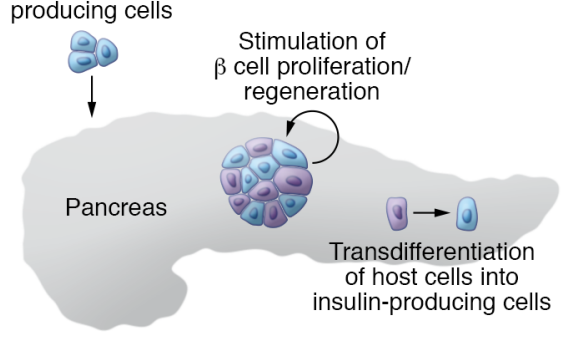

C Protection/immunomodulation

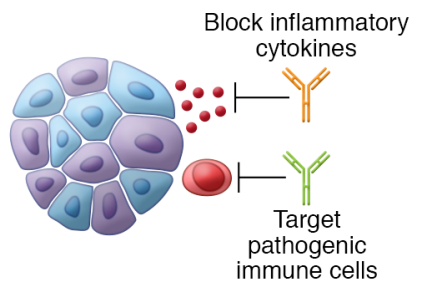

Figure 3. Emerging or future T1D therapies. (A) Exogenous insulin replacement includes insulin analogs designed to optimize absorption, integrated closed-loop systems combining insulin delivery devices and glucose-sensing technology, and personalized algorithms (Al, artificial intelligence) to tailor insulin replacement. (B) Cell-based insulin delivery options include transplantation of islets or insulin-producing cells (derived from ES or iPS cells), strategies to stimulate $\beta$ cell proliferation or regeneration, and approaches that encourage transdifferentiation of host cells into insulin-producing cells. (C) Protective strategies include immunomodulatory approaches to block inflammatory cytokines or pathogenic immune cells and prevent damage or loss of $\beta$ cells. See text for additional information.

\section{Diabetes-related complications and long-term T1D outcomes}

Diabetes-related complications, both microvascular (retinopathy, nephropathy, and neuropathy) and macrovascular (cardiovascular disease, peripheral artery disease), are responsible for the considerable morbidity and mortality in T1D. Although hyperglycemia duration and severity are the drivers of diabetes-related complications in T1D, the molecular mechanisms of how excess glucose leads to a specific organ dysfunction are incompletely defined and likely distinctive for the affected organ system. Diabetes-related complications are likely multifactorial and involve genetic susceptibility to glucose exposure, epigenetic changes induced by hyperglycemia, associated dyslipidemia, and cellular pathways such as advanced glycation end products, sphingolipid metabolism (neuropathy), cytokines, multiple growth factors (VEGF in retinopathy), and/or oxidative stress (111-114). Prevention of diabetesrelated complications by intensive glycemic control is a major focus of T1D clinical care but is challenging because the complications occur years or decades after T1D onset. Improved glycemic control does not reverse established diabetes-related complications.

Long-term outcomes in individuals with T1D have greatly improved with reduced frequency of retinopathy, nephropathy, and neuropathy and improved cardiovascular outcomes; it is becoming increasingly common for individuals to live for more than 50 years with T1D (115). This is partly due to improvements in glycemic control as demonstrated in the Diabetes Control and Complications Trial (DCCT) (116) and partly because of improvements in lipid and blood pressure management or treatments that reduce or delay nephropathy or retinopathy. The DCCT also demonstrated that intensive glycemic control (near normoglycemia) begun at T1D onset partially preserves $\beta$ cell function (and presumably $\beta$ cell mass) as reflected by C-peptide secretion, subsequently leading to improved glycemic control and less hypoglycemia. However, hypoglycemia is a major adverse effect of intensive glycemic control (increased 3-fold in DCCT) and a substantial, lifelong burden for individuals with T1D (99). Individu- als with T1D using intensive glycemic therapy also gained more weight. In the years after the intensive glycemic control phase of the DCCT, study participants in the intense glycemic or control groups were followed as part of the Epidemiology of Diabetes Interventions and Complications (EDIC) study. Both groups in this next phase had similar glycemic control (A1C 8\%), but strikingly, the incidence of complications between the intense treatment groups further widened in the two groups, suggesting that the period of improved glycemic control during the intensive treatment phase translated into a continued and sustained reduction in microvascular complications (116). Termed "metabolic memory," this also translated into improvement in cardiovascular outcomes (117-119). Although some have disputed the concept of metabolic memory (120), it seems likely that early glycemic control has long-term benefits, perhaps lasting more than a decade, maybe through epigenetic mechanisms (116). The clear clinical implication is that glycemic control as close to normoglycemia as safely possible should be the goal beginning immediately after the onset of hyperglycemia.

The management of T1D-related nephropathy, retinopathy, neuropathy, and cardiovascular disease continues to improve with clear benefits of ACE inhibitors and angiotensin receptor blockers in slowing the decline in glomerular filtration rate, anti-VEGF therapies affecting diabetic macular edema and retinopathy, and possibly sodium-glucose cotransporter-2 (SGLT-2) inhibitors or GLP-1 receptor agonists in T1D-related cardiovascular disease $(111,121,122)$. Still, some individuals develop debilitating or life-threatening complications such as end-stage renal disease or neuropathy (122-125). Although intense glycemic control clearly reduces microvascular complications, other factors, such as genetic susceptibility, residual insulin secretion, activity of selected glycolytic enzymes, advanced glycation end-product production, and degree of activation of the renin-angiotensinaldosterone system, may explain the difference in the rates of complications across the T1D population $(115,125)$. A recent estimate predicts a lifespan in individuals with T1D and near-normal 
glycemic control similar to the general population, a remarkable change from past outcomes $(126,127)$.

\section{Emerging and future therapies to treat or prevent T1D}

At this dawn of the next century after insulin's discovery, anticipated new therapies fall into three broad categories (Figure 3): (a) exogenous insulin replacement; (b) cell-based insulin delivery from new sources of insulin-producing cells; and (c) protection of endogenous $\beta$ cells by immunomodulation.

The combination of a glucose sensor and an automatically adjusting insulin delivery device in a closed loop system, often imprecisely termed an "artificial pancreas," is very effective in T1D in children, adults, and pregnancy $(93,128-131)$. This hardware/software is being further improved by emerging algorithms to "correct" interstitial glucose to the actual blood glucose and to predict insulin dosing based on artificial intelligence-based interpretations of personalized glucose excursions and activity. Future improvements in sensor technology may include measuring glucose at other body sites (e.g., eye, skin) or optical/vascular approaches to assess blood rather than interstitial glucose. Improvements in insulin administration are needed as subcutaneous insulin absorption into the vascular system is considerably slower than physiological insulin secretion even with modified insulin analogs (92). Plus, insulin, delivered peripherally and not into the portal vein, leads to reduced insulin action at the liver; it remains unclear how close to "normal" glucose homeostasis can be achieved by insulin delivery at a peripheral site, which also induces insulin resistance as an additional challenge (132). Devices that also deliver glucagon may be part of future therapies (133).

Cell-based insulin delivery may include transplantation of insulin-producing cells, transdifferentiation of other pancreatic cell types (exocrine, ductal, or $\alpha$ ) into insulin-producing cells (134, 135), or amplification/regeneration of endogenous $\beta$ cells (136, 137) (Figure 3). The combination of islet allotransplantation, normal human islets from cadaveric donor(s) infused into the portal vein, and immunosuppression to prevent allo- and autoimmunity is effective in reducing hypoglycemia frequency in T1D, sometimes leading to independence from exogenous insulin (138-142). Because human islet supply is quite limited, insulin-producing cells from embryonic stem cells (ES cells), human induced pluripotent stem cells (iPS cells), or even xenografts (porcine) are also under investigation (139, 143-148). Considerable progress in ES and iPS cell differentiation into insulin-producing cells will soon lead to clinical trials and offer the future possibility of transplanting insulin-producing cells modified to resist allo- and autoimmunity. The optimal site for transplantation of islets or insulin-producing cells is uncertain. Transdifferentiation and regeneration strategies, although attractive, need more research before testing in humans. Glucose- and nutrient-regulated insulin secretion from these new sources of insulin-producing cells is incompletely defined, but crucial. Particularly critical is the appropriate cessation of insulin secretion when the blood glucose is in the normal range to avoid hypoglycemia.

A number of studies, often part of TrialNet (149) or the Immune Tolerance Network (150), suggest that survival of endogenous $\beta$ cells remaining in early stage 3 T1D can be enhanced and partially protected by immunomodulatory approaches directed at B lymphocytes, T lymphocytes, regulatory $\mathrm{T}$ cells, or inflammation by anti-CD3, anti-CD20, CTLA-4Ig, anti-CD2, anti-IL-1, anti-TNF- $\alpha$, or thymoglobulin $(39,151-153)$. However, thus far, such immunomodulatory interventions given soon after hyperglycemia onset have had the modest impact of preserving C-peptide secretion, which is not often sustained. Efforts are underway to understand why only some individuals respond to such therapies, if repeat dosing is needed, and if antigen-specific therapy or exposure (insulin, GAD) can reduce the autoimmune response $(39,154)$. A single course of an Fc receptor-nonbinding anti-CD3 monoclonal antibody given to first-degree relatives at high risk for developing T1D (stage 2, two islet-directed autoantibodies) delayed the onset of hyperglycemia by 2 years, with twice as many individuals progressing to clinical diabetes in the control group (57\% versus $28 \%$ ) (155). This intervention is thought to target "pathogenic" $\mathrm{T}$ cells, raising the possibility of induction of immune tolerance or a reset of the immune system (156). This study also highlights how current efforts in the United States, Europe, and Australia to identify high-risk individuals by screening the general population for T1D-GRS and/or islet-directed autoantibodies may lead to new efforts to prevent T1D (154). Efforts to prevent T1D would also be greatly accelerated by identification of the triggering event(s) for $\beta$ cell-directed autoimmunity.

Over the next decade, it truly will be a competition between these three clinically viable therapeutic options for T1D. For illustrative purposes, consider these scenarios in which glycemia is essentially normalized and diabetes-related complications are prevented by the following: (a) a mechanical insulin-delivery device/ glucose sensor system, requiring little input from the patient and with little limitation of daily diet or activities; (b) transplantation of insulin-producing cells every 1-2 years, accompanied by continuous, "safe" immunomodulation/immunosuppression; and (c) intermittent, immunomodulation/immunosuppression beginning at the onset of hyperglycemia or prior to hyperglycemia (stage 2).

What will be the therapy for T1D in 5 years, 10 years, or 20 years? This will be determined by balancing the benefit, sustainability, adverse impact, safety, convenience, personal preference, and financial cost of these evolving options $(39,157-159)$. Plus, as therapies in each category continue to improve, the "bar is raised" for other competing therapies and the optimal therapy will likely change. In addition, an individual's age and T1D duration will be important considerations in choosing among competing therapies - the choice will likely be different in a 12-year-old with new-onset T1D and a 42-year-old with T1D for 30 years.

In summary, since insulin's discovery, there has been much progress in understanding the pathogenesis of T1D and in using insulin to improve the lives of individuals with T1D. These efforts are incomplete in that T1D continues to be a substantial burden for individuals with T1D and their families. Hopefully, the opportunities discussed here will be realized, leading to the prevention of T1D and its associated burdens.

\section{Acknowledgments}

The author apologizes to the many investigators whose important work on T1D was not cited because of space limitations. Research by the author and his colleagues is or has been supported by the 
National Institute of Diabetes and Digestive and Kidney Diseases; the Human Islet Research Network (RRID: SCR_014393; https:// hirnetwork.org; DK112232, DK123716, DK123743, DK104211, DK108120); and by DK106755, DK117147, DK20593 (Vanderbilt Diabetes Research and Training Center); the Leona M. and Harry B. Helmsley Charitable Trust; the Juvenile Diabetes Research Foundation; and the Department of Veterans Affairs (BX000666).
The author thanks Vanderbilt colleagues, Daniel J. Moore, John T. Walker, and Jordan J. Wright, for reading the manuscript and providing insightful suggestions.

Address correspondence to: Alvin C. Powers, Vanderbilt University Medical Center, 8435 MRBIV, 2215 Garland Ave, Nashville, Tennessee 37232, USA. Phone: 615.936.7678; Email: al.powers@vumc.org.
1. Bliss M. The Discovery of Insulin. University of Toronto Press; 1982.

2. Cooper T, Ainsberg A. Breakthrough: Elizabeth Hughes, the Discovery of Insulin, and the Making of a Medical Miracle. St. Martin's Publishing Group; 2010.

3. Redondo MJ, et al. The clinical consequences of heterogeneity within and between different diabetes types. Diabetologia. 2020;63(10):2040-2048.

4. Balasubramanyam A. Defining and classifying new subgroups of diabetes. Annu Rev Med. 2021;72:1-74.

5. Chung WK, et al. Precision medicine in diabetes: a consensus report from the American Diabetes Association (ADA) and the European Association for the Study of Diabetes (EASD). Diabetologia. 2020;63(9):1671-1693.

6. Atkinson MA, et al. Type 1 diabetes. Lancet. 2014;383(9911):69-82.

7. Insel RA, et al. Staging presymptomatic type 1 diabetes: a scientific statement of JDRF, the Endocrine Society, and the American Diabetes Association. Diabetes Care. 2015;38(10):1964-1974.

8 . Sherry NA, et al. Natural history of $\beta$-cell function in type 1 diabetes. Diabetes. 2005;54(suppl 2):S32-S39.

9. Mäkimattila S, et al. Every fifth individual with type 1 diabetes suffers from an additional autoimmune disease: a Finnish nationwide study. Diabetes Care. 2020;43(5):1041-1047.

10. Center for Disease Control and Prevention. National Diabetes Statistics Report, 2020. https://www.cdc.gov/diabetes/library/features/ diabetes-stat-report.html. Updated February 11, 2020. Accessed March 10, 2021.

11. Maahs DM, et al. Epidemiology of type 1 diabetes. Endocrin Metab Clin. 2010;39(3):481-497.

12. Weng J, et al. Incidence of type 1 diabetes in China, 2010-13: population based study. BMJ. 2017;360:j5295.

13. Harjutsalo V, et al. Incidence of type 1 diabetes in Finland. JAMA. 2013;310(4):427-428.

14. Mayer-Davis EJ, et al. Incidence trends of type 1 and type 2 diabetes among youths, 2002-2012. New Engl J Med. 2017;376(15):1419-1429.

15. Redondo MJ, et al. Genetics of type 1 diabetes. Pediatr Diabetes. 2018;19(3):346-353.

16. Redondo MJ, et al. Concordance for islet autoimmunity among monozygotic twins. N Engl JMed. 2008;359(26):2849-2850.

17. Grant SFA, et al. Next steps in the identification of gene targets for type 1 diabetes. Diabetologia. 2020;63(11):2260-2269.

18. Ferrat LA, et al. A combined risk score enhances prediction of type 1 diabetes among susceptible children. Nat Med. 2020;26(8):1247-1255.

19. Thomas NJ, et al. Frequency and phenotype of type 1 diabetes in the first six decades of life: a cross-sectional, genetically stratified survival analysis from UK Biobank. Lancet Diabetes Endocrinol. 2018;6(2):122-129.

20. Vatanen T, et al. Variation in microbiome LPS immunogenicity contributes to autoimmunity in humans. Cell. 2016;165(4):842-853.

21. Vatanen T, et al. Genomic variation and strain-specific functional adaptation in the human gut microbiome during early life. Nat Microbiol. 2019;4(3):470-479.

22. Liu $X$, et al. Distinct growth phases in early life associated with the risk of type 1 diabetes: the TEDDY study. Diabetes Care. 2020;43(3):556-562.

23. Vehik K, et al. Prospective virome analyses in young children at increased genetic risk for type 1 diabetes. Nat Med. 2019;25(12):1865-1872.

24. de Goffau MC, et al. Fecal microbiota composition differs between children with $\beta$-cell autoimmunity and those without. Diabetes. 2012;62(4):1238-1244.

25. Coppieters KT, et al. Demonstration of isletautoreactive CD8 T cells in insulitic lesions from recent onset and long-term type 1 diabetes patients. JExp Med. 2012;209(1):51-60.

26. Delong T, et al. Pathogenic CD4 T cells in type 1 diabetes recognize epitopes formed by peptide fusion. Science. 2016;351(6274):711-714.

27. Peters $\mathrm{L}$, et al. Islet-immune interactions in type 1 diabetes: the nexus of beta cell destruction. Clin Exp Immunol. 2019;198(3):326-340.

28. Babon JAB, et al. Analysis of self-antigen specificity of islet-infiltrating $\mathrm{T}$ cells from human donors with type 1 diabetes. Nat Med.2016;22(12):1482-1487.

29. Carr ALJ, et al. Histological validation of a type 1 diabetes clinical diagnostic model for classification of diabetes. Diabet Med. 2020;37(12):2160-2168.

30. Campbell-Thompson ML, et al. The influence of type 1 diabetes on pancreatic weight. Diabetologia. 2016;59(1):217-221.

31. Campbell-Thompson ML, et al. The diagnosis of insulitis in human type 1 diabetes. Diabetologia. 2013;56(11):2541-2543.

32. Russell MA, et al. HLA class II antigen processing and presentation pathway components demonstrated by transcriptome and protein analyses of islet $\beta$-cells from donors with type 1 diabetes. Diabetes. 2019;68(5):988-1001.

33. Richardson SJ, et al. Islet cell hyperexpression of HLA class I antigens: a defining feature in type 1 diabetes. Diabetologia. 2016;59(11):2448-2458.

34. Foulis AK, et al. The histopathology of the pancreas in type 1 (insulin-dependent) diabetes mellitus: a 25-year review of deaths in patients under 20 years of age in the United Kingdom. Diabetologia. 1986;29(5):267-274.

35. Oram RA, et al. Beta cells in type 1 diabetes: mass and function; sleeping or dead? Diabetologia. 2019;62(4):567-577.
36. Jacobsen LM, et al. The risk of progression to type 1 diabetes is highly variable in individuals with multiple autoantibodies following screening. Diabetologia. 2020;63(3):588-596.

37. Ziegler AG, et al. Seroconversion to multiple islet autoantibodies and risk of progression to diabetes in children. JAMA. 2013;309(23):2473-2479.

38. Krischer JP, et al. The 6 year incidence of diabetes-associated autoantibodies in genetically at-risk children: the TEDDY study. Diabetologia. 2015;58(5):980-987.

39. Warshauer JT, et al. New frontiers in the treatment of type 1 diabetes. Cell Metab. 2019;31(1):46-61.

40. Michels AW, et al. Islet-derived CD4 T cells targeting proinsulin in human autoimmune diabetes. Diabetes. 2016;66(3):722-734.

41. Mitchell AM, et al. T-cell responses to hybrid insulin peptides prior to type 1 diabetes development. Proc Natl Acad Sci U S A. 2021;118(6):e2019129118.

42. Leete $\mathrm{P}$, et al. Differential insulitic profiles determine the extent of $\beta$-cell destruction and the age at onset of type 1 diabetes. Diabetes. 2016;65(5):1362-1369.

43. Walker JT, et al. The human islet: mini-organ with mega-impact. Endocr Rev. In press.

44. Atkinson MA, et al. Current concepts on the pathogenesis of type 1 diabetes-considerations for attempts to prevent and reverse the disease. Diabetes Care. 2015;38(6):979-988.

45. Atkinson MA, et al. How does type 1 diabetes develop?: the notion of homicide or $\beta$-cell suicide revisited. Diabetes. 2011;60(5):1370-1379.

46. Roep BO, et al. Type 1 diabetes mellitus as a disease of the $\beta$-cell (do not blame the immune system?). Nat Rev Endocrinol. 2020;17(3):150-161.

47. Mallone R, Eizirik DL. Presumption of innocence for beta cells: why are they vulnerable autoimmune targets in type 1 diabetes? Diabetologia. 2020;63(10):1999-2006.

48. Rodriguez-Calvo $\mathrm{T}$, et al. Increase in pancreatic proinsulin and preservation of $\beta$-cell mass in autoantibody-positive donors prior to type 1 diabetes onset. Diabetes. 2017;66(5):1334-1345.

49. Leete $\mathrm{P}$, et al. Studies of insulin and proinsulin in pancreas and serum support the existence of aetiopathological endotypes of type 1 diabetes associated with age at diagnosis. Diabetologia. 2020;63(6):1258-1267.

50. Sims EK, et al. The role of beta-cell dysfunction in early type 1 diabetes. Curr Opin Endocrinol Diabetes Obes. 2020;27(4):215-224.

51. Sims EK, et al. Biomarkers of islet beta cell stress and death in type 1 diabetes. Diabetologia. 2018;61(11):2259-2265.

52. JDRF. Network of Pancreatic Organs for Donors with Diabetes. https://www.jdrfnpod.org/. 
Accessed March 10, 2021.

53. Kaestner KH, et al. NIH initiative to improve understanding of the pancreas, islet, and autoimmunity in type 1 diabetes: the Human Pancreas Analysis Program (HPAP). Diabetes. 2019;68(7):1394-1402.

54. Damond N, et al. A map of human type 1 diabetes progression by imaging mass cytometry. Cell Metab. 2019;29(3):755-768.

55. Wang YJ, et al. Multiplexed in situ imaging mass cytometry analysis of the human endocrine pancreas and immune system in type 1 diabetes. Cell Metab. 2019;29(3):769-783.

56 . Lam CJ, et al. $\beta$ Cells persist in T1D pancreata without evidence of ongoing $\beta$-cell turnover or neogenesis. J Clin Endocrinol Metab. 2017;102(8):2647-2659.

57. Keenan HA, et al. Residual insulin production and pancreatic $\beta$-cell turnover after 50 years of diabetes: Joslin Medalist Study. Diabetes. 2010;59(11):2846-2853.

58. Oram RA, et al. The majority of patients with long-duration type 1 diabetes are insulin microsecretors and have functioning beta cells. Diabetologia. 2013;57(1):187-191.

59. Brissova M, et al. $\alpha$ Cell function and gene expression are compromised in type 1 diabetes. Cell Rep. 2018;22(10):2667-2676.

60. Cryer PE. Hypoglycemia in type 1 diabetes mellitus. Endocrin Metab Clin North Am. 2010;39(3):641-654.

61. Alexandre-Heymann L, et al. Structure and function of the exocrine pancreas in patients with type 1 diabetes. Rev Endocr Metab Disord. 2019;20(2):129-149.

62. Campbell-Thompson ML, et al. Relative pancreas volume is reduced in first-degree relatives of patients with type 1 diabetes. Diabetes Care. 2019;42(2):dc181512-287.

63. Foster TP, et al. Exocrine pancreas dysfunction in type 1 diabetes. Endocr Pract. 2020;26(12):1505-1513.

64. Virostko J, et al. Pancreas volume declines during the first year after diagnosis of type 1 diabetes and exhibits altered diffusion at disease onset. Diabetes Care. 2019;42(2):248-257.

65. Wright JJ, et al. Decreased pancreatic acinar cell number in type 1 diabetes. Diabetologia. 2020;63(7):1418-1423.

66. Bender C, et al. The healthy exocrine pancreas contains preproinsulin-specific CD8 T cells that attack islets in type 1 diabetes. Sci Adv. 2020;6(42):eabc5586.

67. Rodriguez-Calvo $\mathrm{T}$, et al. Increased immune cell infiltration of the exocrine pancreas: a possible contribution to the pathogenesis of type 1 diabetes. Diabetes. 2014;63(11):3880-3890.

68. Skog O, et al. Revisiting the notion of type 1 diabetes being a T-cell-mediated autoimmune disease. Curr Opin Endocrinol Diabetes Obes. 2013;20(2):118-123.

69. Korsgren S, et al. On the etiology of type 1 diabetes: a new animal model signifying a decisive role for bacteria eliciting an adverse innate immunity response. Am J Pathol. 2012;181(5):1735-1748.

70. Battaglia $\mathrm{M}$, et al. Introducing the endotype concept to address the challenge of disease heterogeneity in type 1 diabetes. Diabetes Care.
2019;43(1):5-12.

71. Johnson MB, et al. Monogenic autoimmune diseases of the endocrine system. Lancet Diabetes Endocrinol. 2016;4(10):862-872.

72. Chaimowitz NS, et al. STAT1 gain of function, type 1 diabetes, and reversal with JAK inhibition. N Engl J Med. 2020;383(15):1494-1496.

73. Hattersley AT, Patel KA. Precision diabetes: learning from monogenic diabetes. Diabetologia. 2017;60(5):769-777.

74. Haliyur R, et al. Human islets expressing HNF1A variant have defective $\beta$ cell transcriptional regulatory networks. J Clin Invest. 2018;129(1):246-251.

75. Quandt Z, et al. Autoimmune endocrinopathies: an emerging complication of immune checkpoint inhibitors. Annu Rev Med. 2020;72(1):1-330.

76. Achenbach P, et al. Mature high-affinity immune responses to (pro)insulin anticipate the autoimmune cascade that leads to type 1 diabetes. J Clin Invest. 2004;114(4):589-597.

77. Sosenko JM, et al. The metabolic progression to type 1 diabetes as indicated by serial oral glucose tolerance testing in the Diabetes Prevention Trialtype 1. Diabetes. 2012;61(6):1331-1337.

78. Herold KC, et al. $\beta$ Cell death and dysfunction during type 1 diabetes development in at-risk individuals. JClin Invest. 2015;125(3):1163-1173.

79. Evans-Molina C, et al. $\beta$ Cell dysfunction exists more than 5 years before type 1 diabetes diagnosis. JCI Insight. 2018;3(15):e120877.

80. Vecchio F, et al. Abnormal neutrophil signature in the blood and pancreas of presymptomatic and symptomatic type 1 diabetes. JCI Insight. 2018;3(18):122146.

81. Kallionpaa $\mathrm{H}$, et al. Innate immune activity is detected prior to seroconversion in children with HLA-conferred type 1 diabetes susceptibility. Diabetes. 2014;63(7):2402-2414.

82. Cabrera SM, et al. Blood-based signatures in type 1 diabetes. Diabetologia. 2016;59(3):414-425.

83. Edner NM, et al. Follicular helper $\mathrm{T}$ cell profiles predict response to costimulation blockade in type 1 diabetes. Nat Immunol. 2020;21(10):1244-1255.

84. Kenefeck R, et al. Follicular helper T cell signature in type 1 diabetes. JClin Invest. 2015;125(1):292-303.

85. Campbell-Thompson M, et al. Insulitis and $\beta$-cell mass in the natural history of type 1 diabetes. Diabetes. 2015;65(3):719-731.

86. Nigi L, et al. From immunohistological to anatomical alterations of human pancreas in type 1 diabetes: new concepts on the stage. Diabetes Metab Res Rev. 2020;36(4):e3264.

87. Carlsson S. Etiology and pathogenesis of latent autoimmune diabetes in adults (LADA) compared to type 2 diabetes. Front Physiol. 2019;10:320.

88. Kumar A, Leiva A. Latent autoimmune diabetes in adults (LADA) in Asian and European populations. Diabetes Metab Res Rev. 2017;33(5):e2890.

89. Mishra R, et al. A global perspective of latent autoimmune diabetes in adults. Trends Endocrinol Metab. 2018;29(9):638-650.

90. Andersen MK. New insights into the genetics of latent autoimmune diabetes in adults. Curr Diab Rep. 2020;20(9):43.

91. Ashrafzadeh S, Hamdy O. Patient-driven diabetes care of the future in the technology era. Cell
Metab. 2018;29(3):564-575.

92. Hirsch IB, et al. The evolution of insulin and how it informs therapy and treatment choices. Endocr Rev. 2020;41(5):bnaa015.

93. Beck RW, et al. Advances in technology for management of type 1 diabetes. Lancet. 2019;394(10205):1265-1273.

94. Kesavadev J, et al. Evolution of insulin delivery devices: from syringes, pens, and pumps to DIY artificial pancreas. Diabetes Ther. 2020;11(6):1251-1269.

95. Dovc K, Battelino T. Evolution of diabetes technology. Endocrinol Metab Clin North Am. 2020;49(1):1-18.

96. Maiorino MI, et al. Effects of continuous glucose monitoring on metrics of glycemic control in diabetes: a systematic review with meta-analysis of randomized controlled trials. Diabetes Care. 2020;43(5):1146-1156.

97. American Diabetes Association. 7. Diabetes technology: standards of medical care in diabetes-2021. Diabetes Care. 2021; 44(suppl 1):S85-S99.

98. American Diabetes Association. 6. Glycemic targets: standards of medical care in diabetes-2021. Diabetes Care. 2020;44(suppl 1):S73-S84.

99. Gubitosi-Klug RA, et al. Risk of severe hypoglycemia in type 1 diabetes over 30 years of followup in the DCCT/EDIC study. Diabetes Care. 2017;40(8):1010-1016.

100.Peters AL. Integration of diabetes technology in clinical practice. Endocrinol Metab Clin North Am. 2020;49(1):69-77.

101. Rosenstock J, et al. Once-weekly insulin for type 2 diabetes without previous insulin treatment. NEngl JMed. 2020;383(22):2107-2116.

102. Steiner DF. The Banting Memorial Lecture 1976. Insulin today. Diabetes. 1977;26(4):322-340.

103. Liu M, et al. Biosynthesis, structure, and folding of the insulin precursor protein. Diabetes Obes Metab. 2018;20:28-50.

104. Rege NK, et al. Evolution of insulin at the edge of foldability and its medical implications. Proc Natl Acad Sci U S A. 2020;117(47):29618-29628.

105. Jarosinski MA, et al. 'Smart' insulin-delivery technologies and intrinsic glucose-responsive insulin analogues. Diabetologia. 2021;64(5):1016-1029.

106.Cefalu WT, et al. Insulin access and affordability working group: conclusions and recommendations. Diabetes Care. 2018;41(6):1299-1311.

107. American Diabetes Association. https:// insulinhelp.org/.

108. Carlson $\mathrm{AL}$, et al. $\mathrm{HbA}_{1 \mathrm{c}}$ : the glucose management indicator, time in range, and standardization of continuous glucose monitoring reports in clinical practice. Endocrinol Metab Clin North Am. 2020;49(1):95-107.

109. Galindo RJ, Aleppo G. Continuous glucose monitoring: the achievement of 100 years of innovation in diabetes technology. Diabetes Res Clin Pract. 2020;170:108502.

110. Peters AL. Integration of diabetes technology in clinical practice. Endocrinol Metab Clin North Am. 2019;49(1):69-77.

111. Jampol LM, et al. Evaluation and care of patients with diabetic retinopathy. $\mathrm{N}$ EnglJ Med. 2020;382(17):1629-1637. 
112. Callaghan BC, et al. Diabetic neuropathy: what does the future hold? Diabetologia. 2020;63(5):891-897.

113. Kato M, Natarajan R. Epigenetics and epigenomics in diabetic kidney disease and metabolic memory. Nat Rev Nephrol. 2019;15(6):327-345.

114. Ruiz HH, et al. Advanced glycation end products: building on the concept of the "common soil" in metabolic disease. Endocrinology. 2019;161(1):bqz006.

115. Perkins BA, et al. Discoveries from the study of longstanding type 1 diabetes [published online March 4, 2021]. Diabetologia. https://doi. org/10.1007/s00125-021-05403-9.

116. Nathan DM, et al. Diabetes control and complications trial/epidemiology of diabetes interventions and complications study at 30 years: advances and contributions. Diabetes. 2013;62(12):3976-3986

117. Nathan DM, et al. Intensive diabetes treatment and cardiovascular disease in patients with type 1 diabetes. N Engl J Med. 2005;353(25):2643-2653.

118. Writing Group for the DCCT/EDIC Research Group, et al. Association between 7 years of intensive treatment of type 1 diabetes and longterm mortality. JAMA. 2015;313(1):45-53.

119. Rawshani A, et al. Mortality and cardiovascular disease in type 1 and type 2 diabetes. $N$ Engl J Med. 2017;376(15):1407-1418.

120. Miller RG, Orchard TJ. Understanding metabolic memory: a tale of two studies. Diabetes. 2020;69(3):291-299.

121. American Diabetes Association. 10. Cardiovascular disease and risk management: standards of medical care in diabetes-2021. Diabetes Care. 2021;44(suppl 1):S125-S150.

122. American Diabetes Association. 11. Microvascular complications and foot care: standards of medical care in diabetes. Diabetes Care. 2021;44(suppl 1):S151-S167.

123. Mizokami-Stout KR, et al. The contemporary prevalence of diabetic neuropathy in type 1 diabetes: findings from the T1D exchange. Diabetes Care. 2020;43(4):806-812.

124.Pop-Busui R, et al. Diabetic neuropathy: a position statement by the American Diabetes Association. Diabetes Care. 2016;40(1):136-154.

125. Sandholm N, et al. The genetic landscape of renal complications in type 1 diabetes. J Am Soc Nephrol. 2017;28(2):557-574.

126. Lind M, et al. Glycemic control and excess mortality in type 1 diabetes. $N$ Engl J Med. 2014;371(21):1972-1982.

127. Diabetes Control Complications Trial (DCCT)/
Epidemiology of Diabetes Interventions Complications (EDIC) Study Research Group. Mortality in type 1 diabetes in the DCCT/EDIC versus the general population. Diabetes Care. 2016;39(8):1378-1383.

128. Breton $\mathrm{MD}$, et al. A randomized trial of closedloop control in children with type 1 diabetes. N Engl J Med. 2020;383(9):836-845.

129. Bergenstal RM, et al. Threshold-based insulinpump interruption for reduction of hypoglycemia. N Engl J Med. 2013;369(3):224-232.

130. Bruttomesso D. Toward automated insulin delivery. N Engl J Med. 2019;381(18):1774-1775.

131. Boughton CK, Hovorka R. New closed-loop insulin systems [published online February 6, 2021]. Diabetologia. https://doi.org/10.1007/s00125021-05391-w.

132. Gregory JM, et al. Iatrogenic hyperinsulinemia, not hyperglycemia, drives insulin resistance in type 1 diabetes as revealed by comparison with GCK-MODY (MODY2). Diabetes. 2019;68(8):db190324.

133. Russell SJ, et al. Outpatient glycemic control with a bionic pancreas in type 1 diabetes. $N$ Engl J Med. 2014;371(4):313-325.

134. Furuyama K, et al. Diabetes relief in mice by glucose-sensing insulin-secreting human $\alpha$-cells Nature. 2019;567(7746):43-48.

135. Chera S, et al. Diabetes recovery by age-dependent conversion of pancreatic $\delta$-cells into insulin producers. Nature. 2014;514(7523):503-507.

136. Wang P, et al. Combined inhibition of DYRK1A, SMAD, and trithorax pathways synergizes to induce robust replication in adult human beta cells. Cell Metab. 2019;29(3):638-652.

137. Aguayo-Mazzucato C, Bonner-Weir S. Pancreatic $\beta$ cell regeneration as a possible therapy for diabetes. Cell Metab. 2017;27(1):57-67.

138. Chang CA, et al. Current issues in allogeneic islet transplantation. Curr Opin Organ Transplant. 2017;22(5):437-443.

139. Bellin MD, Dunn TB. Transplant strategies for type 1 diabetes: whole pancreas, islet and porcine beta cell therapies. Diabetologia. 2020;63(10):2049-2056.

140. Shapiro AM, et al. Islet transplantation in seven patients with type 1 diabetes mellitus using a glucocorticoid-free immunosuppressive regimen. N Engl J Med. 2000;343(4):230 238-238.

141. Hering BJ, et al. Phase 3 trial of transplantation of human islets in type 1 diabetes complicated by severe hypoglycemia. Diabetes Care. 2016;39(7):1230-1240.

142. Rickels MR, Robertson RP. Pancreatic islet trans- plantation in humans: recent progress and future directions. Endocr Rev. 2018;40(2):631-668.

143. Pagliuca FW, et al. Generation of functional human pancreatic $\beta$ cells in vitro. Cell. 2014;159(2):428-439.

144. Rezania A, et al. Reversal of diabetes with insulin-producing cells derived in vitro from human pluripotent stem cells. Nat Biotechnol. 2014;32(11):1121-1133.

145. Russ HA, et al. Controlled induction of human pancreatic progenitors produces functional betalike cells in vitro. EMBO J. 2015;34(13):1759-1772.

146. Nair GG, et al. Emerging routes to the generation of functional $\beta$-cells for diabetes mellitus cell therapy. Nat Rev Endocrinol. 2020;16(9):506-518.

147. Millman JR, et al. Generation of stem cellderived $\beta$-cells from patients with type 1 diabetes. Nat Commun. 2016;7(1):11463.

148.Vantyghem M-C, et al. Advances in $\beta$-cell replacement therapy for the treatment of type 1 diabetes. Lancet. 2019;394(10205):1274-1285.

149. TrialNet. Type 1 Diabetes TrialNet. https://www. trialnet.org/. Accessed March 10, 2021.

150.Immune Tolerance Network. https://www. immunetolerance.org/. Accessed March 10, 2021.

151. Coppieters K, von Herrath M. The development of immunotherapy strategies for the treatment of type 1 diabetes. Front Med (Lausanne) 2018;5:283.

152. Battaglia M, et al. Understanding and preventing type 1 diabetes through the unique working model of TrialNet. Diabetologia. 2017;60(11):2139-2147.

153. Quattrin T, et al. Golimumab and beta-cell function in youth with new-onset type 1 diabetes. N Engl JMed. 2020;383(21):2007-2017.

154. Dayan CM, et al. Changing the landscape for type 1 diabetes: the first step to prevention. Lancet. 2019;394(10205):1286-1296.

155. Herold KC, et al. An anti-CD3 antibody, teplizumab, in relatives at risk for type 1 diabetes. NEngl JMed. 2019;381(7):603-613.

156. Bluestone JA, Anderson M. Tolerance in the age of immunotherapy. $N$ Engl J Med 2020;383(12):1156-1166

157. Latres $\mathrm{E}$, et al. Navigating two roads to glucose normalization in diabetes: automated insulin delivery devices and cell therapy. Cell Metab. 2019;29(3):545-563.

158. Accili D. Whither type 1 diabetes? N Engl J Med. 2020;383(21):2078-2079.

159. Linsley PS, et al. Uncovering pathways to personalized therapies in type 1 diabetes. Diabetes. 2021;70(4):831-841. 